\title{
Care Transitions: It's the How, Not Just the What
}

\author{
Jing Li, M.D., M.S. and Mark V. Williams, M.D. \\ Center for Health Services Research, Department of Internal Medicine, University of Kentucky, Lexington, KY, USA.
}

J Gen Intern Med 30(5):539-40

DOI: $10.1007 / \mathrm{s} 11606-015-3225-6$

(c) Society of General Internal Medicine 2015

I $\mathrm{t}$ is widely recognized that vulnerable patients and caregivers attempting to navigate the transition from hospital to home are likely to encounter a stressful period fraught with a high risk of adverse events, rehospitalization, and often unsuccessful follow-up. ${ }^{1,2}$ Quality improvement specialists and administrators have identified this care transition as a target for improving outcomes, particularly for elderly patients with multiple comorbidities, and as a financial risk for hospitals, given the Hospital Readmissions Reduction Program (HRRP) penalty contained within the Patient Protection and Affordable Care Act. ${ }^{3}$ Not surprisingly, researchers have sought to identify interventions that might reduce the rate of rehospitalization, though they have often targeted specific diseases. ${ }^{4} \mathrm{Sev}-$ eral evidence-based transitional care models or intervention bundles have been shown to reduce 30 -day readmissions, ${ }^{5}$ but research has not delineated which components of these transitional care approaches are necessary at what dose; how implementation of core components may need to be adjusted based on patient, caregiver, care delivery setting, or community/environmental characteristics; or how knowledge regarding core components can be used to accelerate system redesign.

In this issue, Field and colleagues have attempted to determine whether the singular intervention of a primary care physician follow-up visit within 7 days of hospital discharge might reduce readmissions. ${ }^{6}$ While prior large retrospective dataset analyses have documented that such early follow-up was associated with lower rates of rehospitalization among patients with heart failure or $\mathrm{COPD},{ }^{7,8}$ prospective studies are lacking. Unfortunately, Field's prospective observational study showed no apparent benefit. A number of factors may account for the observed lack of effectiveness for a seemingly important intervention for patients discharged from the hospital. Though they applied multivariable proportional hazards models to control for confounding factors in their analyses, and further developed a high-dimensional propensity score, they do not report any assessment of patients' psychosocial characteristics. Additionally, their models appear to lack ade-

Published online February 13, 2015 quate controls for socioeconomic effects. Prior studies strongly link social, behavioral, and community factors with hospital readmissions, even after accounting for possible variability in quality of care in the hospital. ${ }^{9-11}$

Moreover, their study did not assess what actually happened during the outpatient follow-up appointment or prior to it. If busy primary care providers fail to receive adequate communication about their patient's hospitalization (e.g., medication changes, test results needing follow-up, or needed follow-up procedures), or cannot review sufficiently documented discharge summaries by the time of the follow-up appointment, they likely will be unable to make appropriate evaluation and management decisions. ${ }^{12}$ Did medication reconciliation occur? Was there coordination among other providers, arrangements for needed social services, or the capability to reach the primary care provider prior to the appointment with questions regarding potential exacerbation of symptoms? Field's study confirms there is no silver bullet to reduce post-hospital utilization by simply coordinating a patient showing up in their doctor's office within a week of hospital discharge.

Evidence increasingly does show that multi-faceted interventions, unlike singular interventions, can improve patient outcomes and reduce 30-day readmissions. ${ }^{13,14}$ A recent 2014 systematic review and meta-analysis provided objective support for the claim that bundled interventions are of greater value. ${ }^{15}$ This review also showed that interventions using a complex and supportive strategy to assess and address contextual issues and limitations in a patient's self-care capacity were most effective at reducing early hospital readmissions.

Public and private payers view reducing avoidable hospital readmissions as a way to improve quality and reduce unnecessary costs. While policymakers have targeted care processes within hospitals, we also believe that readmissions can be prevented if patients receive appropriate follow-up care and ongoing management of their various chronic illnesses. However, this is a process, not simply a one-time event (such as a single doctor's appointment). Fortunately, since 2011, states can apply for federal funds to pay providers to furnish specified "health home" services for Medicaid recipients with certain chronic diseases. Medicare reimbursement for postdischarge services known as "transitional care management (TCM)" began in 2013 as a method of facilitating services for patients with complex medical needs after hospitalization. And with the publication of the 2015 Medicare Physician Fee Schedule Final Rule, beginning January 1, 2015, Medicare will pay for chronic care management, or CCM, aiming to improve access, communication, and care coordination. All of 
these incentive programs are structured strategies designed to deliver defined, evidence-based services to patients. However, while the implementation of these strategies shows early promise in curbing readmission rates, with possible adoption into widespread practice, ${ }^{16,17}$ we remain uncertain as to exactly what interventions or intervention clusters will change patient-centered outcomes, quality of care, and resource utilization in "real-world" settings.

In our experience, transitional care interventions have a high degree of variability (heterogeneity) across context, content, application, and outcome. Oftentimes, programs must be adapted from their original design to conform to the cultural, historical, or other contexts of a particular setting. Changes might be made to a program to better meet the needs of the setting where it is being implemented, to fit the program within an organization's culture or available resources, or to accommodate the preferences of the local staff members who facilitate it. As such, fidelity (i.e., conformity) to the original validated program may not always occur (either intentionally, due to feasibility constraints or other factors, or inadvertently), and rigid fidelity (rather than adaptation to local circumstances) may not yield optimal outcomes. In transitional care research, it is not sufficient to know the magnitude, severity, and causes of the problem and the relative effectiveness (whether it works) of specific interventions to inform practice; it is also necessary to know how a specific intervention or cluster of interventions should be implemented, for whom, and under which circumstances it can be successful. Based on the hypothesis that the effectiveness of transitional care depends on providing services well aligned with the needs of different patient populations within the context of diverse healthcare and community settings, the Patient-Centered Outcomes Research Institute (PCORI) released the Effectiveness of Transitional Care funding announcement in the spring of 2014. PCORI subsequently awarded a grant to Project ACHIEVE (Achieving Patient-Centered Care and Optimized Health In Care Transitions by Evaluating the Value of Evidence) for studies to determine which transitional care intervention clusters are most successful in improving patient-centered outcomes, for whom, and under what circumstances. ${ }^{18}$

As a result of policy and professional initiatives, transitional care programs have been launched in more than 500 communities across the U.S. However, few studies ${ }^{19}$ have answered important questions such as: What core components were successful in promoting improvements in evidence-based programs, in what environments, and why? How much fidelity to the originally described intervention approach is necessary to ensure effectiveness? What types of appropriate adaptations can be made when implementing locally? How can transformative changes be sustained? Future research needs to report not only on the science of evidence but also the science of implementation, and researchers should describe the intervention and contextual factors in sufficient detail to allow local decision-makers the ability to make their own judgments about the applicability of the findings to their own settings and context.

Corresponding Author: Mark V. Williams, M.D.; Center for Health Services Research, Department of Internal MedicineUniversity of Kentucky, 740 South Limestone, Kentucky Clinic J525, Lexington, KY 40536-0284, USA (e-mail: mark.will@uky.edu).

\section{REFERENCES}

1. Forster AJ, Murff HJ, Peterson JF, Gandhi TK, Bates DW. The incidence and severity of adverse events affecting patients after discharge from the hospital. Ann Intern Med. 2003;138(3): 161-167.

2. Jencks SF, Williams MV, Coleman EA. Rehospitalizations among patients in the medicare fee-for-service program. N Engl J Med. 2009;360(14): 1418-1428.

3. Hospital Readmissions Reduction Program. http://www.cms.gov/Medicare/Medicare-Fee-for-Service-Payment/AcuteInpatientPPS / Readmissions-Reduction-Program.html. Accessed 16 Jan 2015.

4. Williams MV. A requirement to reduce readmissions: take care of the patient, not just the disease. JAMA. 2013;309(4):394-396.

5. Li J, Young R, Williams MV. Optimizing transitions of care to reduce rehospitalizations. Cleve Clin J Med. 2014;81(5):312-320.

6. Field TS, Ogarek J, Garber L, Reed G, Gurwitz JH. Association of early post-discharge follow-up by a primary care physician and 30-day rehospitalization among older adults. JGIM. 2015;[3106]

7. Sharma G, Kuo Yf Fau - Freeman JL, Freeman J1 Fau - Zhang DD, Zhang Dd Fau - Goodwin JS, Goodwin JS. Outpatient follow-up visit and 30-day emergency department visit and readmission in patients hospitalized for chronic obstructive pulmonary disease. 20101012 DCOM20101115 (1538-3679 (Electronic)).

8. Hernandez AF, Greiner Ma Fau - Fonarow GC, Fonarow Gc Fau Hammill BG, et al. Relationship between early physician follow-up and 30-day readmission among medicare beneficiaries hospitalized for heart failure. 20100505 DCOM- 20100506 (1538-3598 (Electronic)).

9. Kind AJ, Jencks S, Brock J, et al. Neighborhood socioeconomic disadvantage and 30-day rehospitalization: a retrospective cohort study. Ann Intern Med. 2014;161(11):765-774.

10. Kangovi S, Barg FK, Carter T, et al. Challenges faced by patients with low socioeconomic status during the post-hospital transition. J Gen Intern Med. 2014;29(2):283-289.

11. Hu J, Gonsahn M, Nerenz D. Socioeconomic status and readmissions: evidence from an urban teaching hospital. Health Aff (Project Hope). 2014;33(5):778-785

12. Kripalani S, LeFevre F, Phillips CO, Williams Mv, Basaviah P, Baker DW. Deficits in communication and information transfer between hospitalbased and primary care physicians: implications for patient safety and continuity of care. JAMA. 2007;297(8):831-841.

13. Hansen LO, Young RS, Hinami $\mathbf{K}$, Leung A, Williams MV. Interventions to reduce 30-day rehospitalization: a systematic review. Ann Intern Med. 2011;155(8):520-528.

14. Rennke S, Nguyen OK, Shoeb MH, Magan Y, Wachter RM, Ranji SR. Hospital-initiated transitional care interventions as a patient safety strategy: a systematic review. Ann Intern Med. 2013;158(5 Pt 2):433-440.

15. Leppin AL, Gionfriddo MR, Kessler M, et al. Preventing 30-day hospital readmissions: a systematic review and meta-analysis of randomized trials. 20140709 DCOM- 20140926 (2168-6114 (Electronic)).

16. Costantino ME, Frey B, Hall B, Painter P. The Influence of a postdischarge intervention on reducing hospital readmissions in a medicare population. Popul Health Manag. 2013;16(5):310-316.

17. Naylor MD, Bowles KH, McCauley KM, et al. High-value transitional care: translation of research into practice. J Eval Clin Pract. 2013; 19(5):727-733.

18. Project ACHIEVE. http://www.pcori.org/research-results/2014/projectachieve-achieving-patient-centered-care-and-optimized-health-care. Accessed 21 Jan 2015.

19. Pronovost P Fau - Jha AK, Jha AK. Did hospital engagement networks actually improve care? 20141121 DCOM- 20141125 (1533-4406 (Electronic)). 